\title{
Motion Analytics of Trapezius Muscle Activity in an 18-Year-Old Female with Extended Upper Brachial Plexus Birth Palsy
}

\author{
Jasmine J. Lin ${ }^{1}$ Gromit Y.Y. Chan ${ }^{2}$ Cláudio T. Silva ${ }^{2}$ \\ Aleksandra McGrath ${ }^{5}$ Alice $\mathrm{Chu}^{1}$ \\ ${ }^{1}$ Department of Orthopaedics, Rutgers New Jersey Medical School, \\ Newark, New Jersey, United States \\ 2 Tandon School of Engineering, New York University, New York, \\ United States \\ ${ }^{3}$ Instituto de Ciências Matemáticas e de Computação (ICMC), \\ University of São Paulo, São Paulo, Brazil \\ ${ }^{4}$ Department of Physical Medicine and Rehabilitation and Neurology, \\ Johns Hopkins University School of Medicine, Baltimore, Maryland, \\ United States \\ ${ }^{5}$ Department of Clinical Sciences, Umeå University, Umeå, Sweden
}

Luis G. Nonato ${ }^{3}$ Preeti Raghavan ${ }^{4}$

Address for correspondence Alice Chu, MD, 140 Bergen Street, D Level, Newark, NJ 07103, United States

(e-mail: chual@njms.rutgers.edu).

J Brachial Plex Peripher Nerve Inj 2021;16:e51-e55.

\begin{abstract}
Background The trapezius muscle is often utilized as a muscle or nerve donor for repairing shoulder function in those with brachial plexus birth palsy (BPBP). To evaluate the native role of the trapezius in the affected limb, we demonstrate use of the Motion Browser, a novel visual analytics system to assess an adolescent with BPBP.

Method An 18-year-old female with extended upper trunk (C5-6-7) BPBP underwent bilateral upper extremity three-dimensional motion analysis with Motion Browser. Surface electromyography (EMG) from eight muscles in each limb which was recorded during six upper extremity movements, distinguishing between upper trapezius (UT) and lower trapezius (LT). The Motion Browser calculated active range of motion (AROM), compiled the EMG data into measures of muscle activity, and displayed the results in charts.

Results All movements, excluding shoulder abduction, had similar AROM in affected and unaffected limbs. In the unaffected limb, LT was more active in proximal movements of shoulder abduction, and shoulder external and internal rotations. In the

Keywords

- brachial plexus

- motion analysis

- birth palsy

- nerve transfer

- trapezius

- motion browser affected limb, LT was more active in distal movements of forearm pronation and supination; UT was more active in shoulder abduction.

Conclusion In this female with BPBP, Motion Browser demonstrated that the native LT in the affected limb contributed to distal movements. Her results suggest that sacrificing her trapezius as a muscle or nerve donor may affect her distal functionality. Clinicians should exercise caution when considering nerve transfers in children with BPBP and consider individualized assessment of functionality before pursuing surgery.
\end{abstract}

received

January 25, 2021

accepted after revision

March 31, 2021
DOI https://doi.org/

$10.1055 / \mathrm{s}-0041-1731748$.

ISSN $1749-7221$. (c) 2021. The Author(s).

This is an open access article published by Thieme under the terms of the Creative Commons Attribution License, permitting unrestricted use, distribution, and reproduction so long as the original work is properly cited. (https://creativecommons.org/licenses/by/4.0/)

Georg Thieme Verlag KG, Rüdigerstraße 14, 70469 Stuttgart, Germany 


\section{Introduction}

For brachial plexus surgeons, there are few areas more intriguing than the dynamics of how children with brachial plexus birth palsy (BPBP) use their affected arm. ${ }^{1-3}$ Functionality of the affected arm is not well understood because the injury occurs in the perinatal period and the disease transforms as the child grows. Nerve and muscle weakness develop over time, as well as changes to muscle, joint, bone, and compensatory muscle recruitment. ${ }^{4}$

One interesting question in children with BPBP is whether the upper or lower trapezius (UT and LT, respectively) muscles are functionally active during upper extremity use. It is intriguing because there are three ways in which an innervated trapezius can be utilized in children with BPBP, leaving it in situ, denervation and use of the trapezius' spinal accessory nerve (SAN) for distal nerve transfers, or using it directly as a muscle transfer. ${ }^{5-7}$ The prevailing surgical technique of treating BPBP addresses a compromised suprascapular nerve through a posterior approach of transferring the lower portion of the SAN which innervates the LT and leaving innervation of the UT intact. ${ }^{8,9}$

Because there are no studies looking at the natural contribution of the trapezius in children with BPBP, the morbidity of harvesting the SAN is largely unknown. It is known that normal children who sustain iatrogenic SAN injury have limited shoulder abduction of 70 to 90 degrees, and that divisions of trapezius muscles each have diverse roles in scapulothoracic movements that contribute to glenohumeral stability. ${ }^{10-12}$

To better understand the functional role of the trapezius in children with BPBP, this report presents pilot data on UT and LT activity in an 18-year-old female with extended upper brachial plexus (C5-6-7) birth palsy. It is the first of its kind to demonstrate use of the Motion Browser, a novel visual analytics system developed at New York University. Motion Browser is a tool that synchronizes video recordings with kinematic and EMG data for assessment of muscle groups. ${ }^{13}$

\section{Methods}

An 18-year-old female with right-sided extended upper trunk (C5-6-7) BPBP, Mallet score of 22, and a prior anterior shoulder release underwent bilateral upper extremity analysis with the Motion Browser. She had no prior nerve transfer surgery and was free of neurological or metabolic impairment beyond BPBP. Examination at time of presentation confirmed intact trapezius function; the only physical limitation was right shoulder motion. She had mild internal shoulder rotation, elbow flexion contracture deformity, and weaker wrist flexion compared with the contralateral side, but was greater than the British Medical Research Council (BMRC), grade 3 in all tested motions. In this institutional review board (IRB) approved study at New York University, the patient was evaluated with simultaneous threedimensional motion analysis, 16-channel electromyography (EMG), and video monitoring.

Data were recorded for eight muscles in each upper limb as follows: (1) biceps, (2) triceps, (3) pronator teres, (4) pronator quadratus, (5) UT, (6) LT, (7) flexor digitorum superficialis, and (8) extensor digitorum communis. The following six upper extremity movements were performed: (1) shoulder abduction, (2) shoulder external rotation, (3) shoulder internal rotation, (4) elbow flexion, (5) forearm pronation, and (6) forearm supination. Movements were tested individually in each limb. Only one recording for each limb was assessed per movement. Shoulder external/internal rotation was performed with the arm in the adducted position. Nonshoulder movements of elbow flexion and forearm pronation/supination were called distal movements. EMG, kinematic data, and video recordings were processed through the Motion Browser. ${ }^{13}$

Data were selected in the Motion Browser, starting from the auditory tone that prompted the patient to initiate motion and ending when the arm returned to the resting position. Twelve-movement segments were collected, six movements per limb. Within each movement, maximal active motion and muscle activity patterns were recorded. - Fig. 1 displays the data extraction steps.

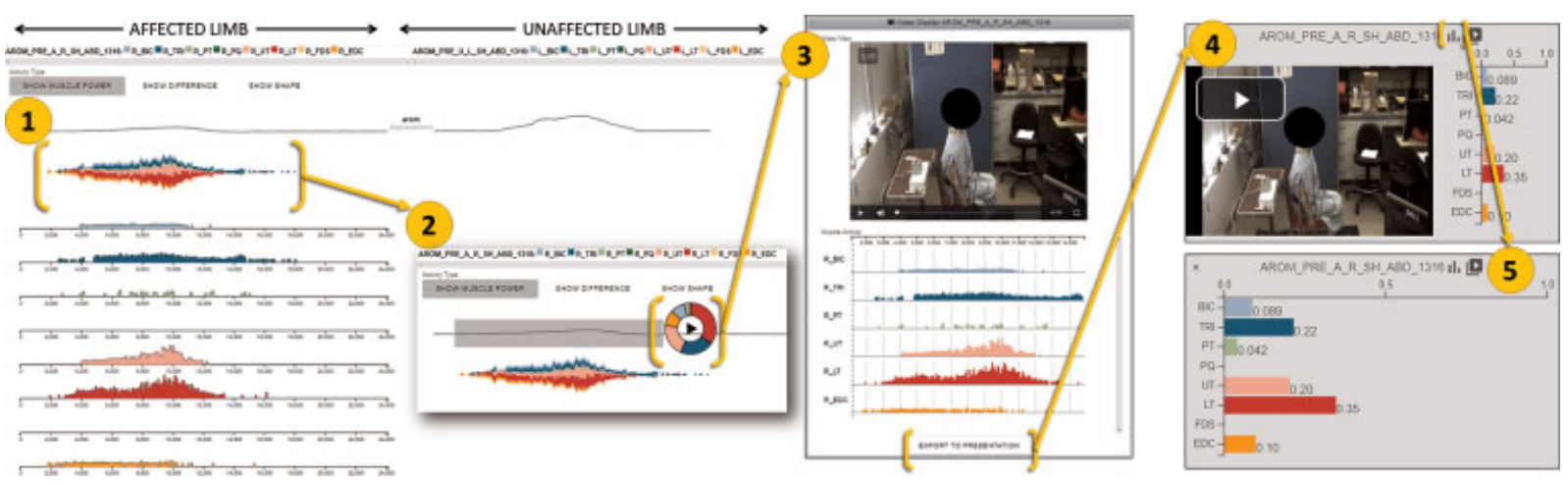

Fig. 1 The Motion Browser data extraction method. 1. The Motion Browser displays the synchronized video, kinematics, and electromyography (EMG) data for the patient's affected and unaffected limbs for each movement performed. 2 . The user selected segments of interest based on examining the video clips to fit selection criteria. Selected segments included all EMG data corresponding to the movement recorded in that video segment. 3. The Motion Browser isolated the motion data for that segment of interest. 4. The Motion Brower displayed a bar chart depicting the fraction of each muscle's activation relative to the total measured activity in the movement in the segment of interest. 5 . Individual bar values for muscle activation were recorded for results analysis. 
The Motion Browser calculated active range of motion (AROM) as the angular displacement (in degrees) between the highest point of upper limb placement and the lowest point of the patient's resting position. Pronation and supination were measured with supination scored as negative. The similarity ratio between the maximum AROM in the affected versus the unaffected limb was calculated as a percentage. If the patient produced more voluntary motion in the affected than in the unaffected limb, a ratio of greater than $100 \%$ was calculated.

The Motion Browser compiled the EMG data throughout the segment into measures of muscle activity by the rootmean-square (RMS) envelope and displayed the results of the eight muscle groups in bar charts. ${ }^{11}$ Each bar value demonstrated the fraction of muscle activity relative to the total measured activity throughout the movement. The higher the bar value, the higher the individual muscle activity. Muscle activity for UT and LT was isolated for assessment.

\section{Results}

\section{Voluntary Active Range of Motion Was Similar in Both Affected and Unaffected Limbs}

Similarity of the voluntary affected/unaffected maximal AROM is shown in - Table 1. All movements excluding shoulder abduction had a similarity ratio (affected/unaffected) greater than or equal to $90 \%$. Only one movement, shoulder abduction, had an affected/unaffected ratio $<90 \%$, at $60.81 \%$.

\section{Lower Trapezius Was More Active in the Affected Limb during Distal Movements}

Trapezius muscle activity shown in -Table 2 displays the percentage of EMG activity contributed by UT and LT in all movements in each limb. UT had no activity in affected or unaffected limbs in shoulder external rotation, shoulder internal rotation, elbow flexion, forearm pronation, and forearm supination. UT accounted for $20.0 \%$ of muscle activ-

Table 1 Results for comparing the ratio of angular displacement of active motion between affected and unaffected limbs in upper extremity movements $(\mathrm{cm})$

\begin{tabular}{|l|l|l|l|}
\hline Movement & Affected & Unaffected & $\begin{array}{l}\text { Similarity } \\
\text { ratio } \\
\text { (affected/ } \\
\text { unaffected) \% }\end{array}$ \\
\hline Shoulder abduction & 74.8 & 123 & 60.81 \\
\hline $\begin{array}{l}\text { Shoulder external } \\
\text { rotation }\end{array}$ & 57.6 & 60.6 & 95.05 \\
\hline $\begin{array}{l}\text { Shoulder internal } \\
\text { rotation }\end{array}$ & 67.4 & 64 & 105.31 \\
\hline Elbow flexion & 162 & 152 & 106.58 \\
\hline Forearm pronation & 128 & 142 & 90.14 \\
\hline Forearm supination & 113 & 123 & 91.87 \\
\hline
\end{tabular}

Table 2 Percentage of EMG activity contributed by the UT and LT in the six tested movements in both affected and unaffected limbs

\begin{tabular}{|c|c|c|}
\hline \multicolumn{3}{|l|}{ UT activity (\%) } \\
\hline Movement & Affected & Unaffected \\
\hline Shoulder abduction & 20.0 & 0.5 \\
\hline Shoulder external rotation & 0.0 & 0.0 \\
\hline Shoulder internal rotation & 0.0 & 0.0 \\
\hline Elbow flexion & 0.0 & 0.0 \\
\hline Forearm pronation & 0.0 & 0.0 \\
\hline Forearm supination & 0.0 & 0.0 \\
\hline \multicolumn{3}{|l|}{ LT activity (\%) } \\
\hline Movement & Affected & Unaffected \\
\hline Shoulder abduction & 35.0 & 85.0 \\
\hline Shoulder external rotation & 6.0 & 30.0 \\
\hline Shoulder internal rotation & 7.9 & 61.0 \\
\hline Elbow flexion & 35.0 & 17.0 \\
\hline Forearm pronation & 59.0 & 7.0 \\
\hline Forearm supination & 87.0 & 23.0 \\
\hline
\end{tabular}

Abbreviations: EMG, electromyography; LT, lower trapezius; UT, upper trapezius.

ity in the affected limb during shoulder abduction and $0.5 \%$ in the unaffected during shoulder abduction.

LT was most active ( $>50 \%$ ) in the unaffected limb during shoulder abduction (85.0\%) and shoulder internal rotation (61.0\%). It was most active ( $>50 \%$ ) in the affected limb during the distal movements of forearm pronation (59.0\%) and forearm supination (87.0\%). Activity in the unaffected limb was more than that in the affected limb during shoulder abduction (85.0\% unaffected and $35.0 \%$ affected), shoulder external rotation (30.0\% unaffected and $6.0 \%$ affected), and shoulder internal rotation (61.0\% unaffected and $8.0 \%$ affected). Activity in the affected limb was more than that in the unaffected during elbow flexion $(35.0 \%$ affected and $17.0 \%$ unaffected), forearm pronation (59.0\% affected and $7.0 \%$ unaffected), and forearm supination (87.0\% affected and $23.0 \%$ unaffected).

\section{Discussion}

The effect of the trapezius muscle, while known to be vital for upper limb function in healthy children, ${ }^{11}$ has never been studied in children/adolescents with BPBP. Over the past decade, studies have discussed the transfer of the trapezius' SAN for a compromised suprascapular nerve in treating BPBP. $^{14-16}$ However, a necessary prerequisite to perform a nerve transfer is the consensus that the donor nerve is expendable and will not downgrade the patient's upper extremity function. ${ }^{17,18}$ Morbidity of trapezius innervation or transfer should first be determined based on knowledge about its function in the natural state of injury. 
Most authors who study upper extremity function in neurological diseases utilize kinematics or combined EMG analysis. ${ }^{18-21}$ The Motion Browser is unique because it expands upon that methodology by synchronizing video recordings with kinematic and EMG data, thus corresponding information at each time point can be isolated. EMG data are normalized by comparing muscle activity within the limb, and the browser displays percentages of each muscle activity. ${ }^{13}$

This patient's Motion Browser results suggest that her LT is more active in the affected limb during the movements of forearm pronation $(59.0 \%)$ and supination $(87.0 \%)$ than in the unaffected (17.0 and $23.0 \%$, respectively). The AROM of the affected limb when compared with the unaffected during these movements had a similarity ratio of $>90.0 \%$. In these movements that are not typically expected to provoke trapezius activity, the muscle likely plays a part in isokinetic stabilization of the scapulothoracic region. ${ }^{10-12}$ Conversely, for UT, activity was nearly zero for all movements in the affected limb except for shoulder abduction (20.0\%) and minimally active in the unaffected for all movements. In the video footage, the patient appeared to lift the affected shoulder to complete shoulder abduction. UT activation can thus be attributed to compensating for the abduction deficit, a sequela of the brachial plexus injury.

This report demonstrates the use of the Motion Browser for analysis of the trapezius muscle in an adolescent with BPBP. This patient's results in the affected limb showed LT active a decreased amount during functional shoulder and elbow motion, but relatively active during the most distal movements of forearm supination and pronation. Results suggested that in this specific patient, transferring the SAN could potentially compromise distal function at the expense of shoulder restoration.

\section{Limitations}

Limitations of the Motion Browser include the constraints of data collection. This setup is expensive because analyses need to be completed in a laboratory with adequate equipment. Furthermore, EMG signals are collected through surface electrodes, which are prone to background noise and difficulty with normalization.

\section{Conclusion}

In conclusion, clinicians may wish to exercise caution when considering the current surgical treatment of the transfer of the SAN for the suprascapular nerve. Studies in patients who did and did not receive nerve transfer have found no significant differences in external rotation of the $\mathrm{arm}^{22}$ or in recovery of ROM or strength ${ }^{23}$; postsurgical follow-ups have found no trapezius atrophy/weakness. ${ }^{24}$ Motion systems, like the Motion Browser, can help clinicians better evaluate the native use of the trapezius muscle in BPBP patients before considering surgery.

\section{Funding}

None.

Conflict of Interest

None declared.

\section{References}

1 Abzug JM, Kozin SH. Evaluation and management of brachial plexus birth palsy. Orthop Clin North Am 2014;45(02):225-232

2 Duff SV, Dayanidhi S, Kozin SH. Asymmetrical shoulder kinematics in children with brachial plexus birth palsy. Clin Biomech (Bristol, Avon) 2007;22(06):630-638

3 Waters PM. Comparison of the natural history, the outcome of microsurgical repair, and the outcome of operative reconstruction in brachial plexus birth palsy. J Bone Joint Surg Am 1999;81 (05):649-659

4 Min W, Price AE, Alfonso I, Ramos L, Grossman JA. Hypoplasia of the trapezius and history of ipsilateral transient neonatal brachial plexus palsy. Pediatr Neurol 2011;44(03):225-228

5 Colbert SH, Mackinnon S. Posterior approach for double nerve transfer for restoration of shoulder function in upper brachial plexus palsy. Hand (N Y) 2006;1(02):71-77

6 Kawabata H, Kawai H, Masatomi T, Yasui N. Accessory nerve neurotization in infants with brachial plexus birth palsy. Microsurgery 1994;15(11):768-772

7 Midha R. Nerve transfers for severe brachial plexus injuries: a review. Neurosurg Focus 2004;16(05):E5

8 Bhandari PS, Sadhotra LP, Bhargava P, Singh M, Mukherjee MK, Bhatoe HS. Dorsal approach in spinal accessory to suprascapular nerve transfer in brachial plexus injuries: technique details. Indian J Neurotrauma 2010;7(01):71-74

9 Segal D, Cornwall R, Little KJ. Outcomes of spinal accessory-tosuprascapular nerve transfers for brachial plexus birth injury. J Hand Surg Am 2019;44(07):578-587

10 Cools AM, Declercq GA, Cambier DC, Mahieu NN, Witvrouw EE. Trapezius activity and intramuscular balance during isokinetic exercise in overhead athletes with impingement symptoms. Scand J Med Sci Sports 2007;17(01):25-33

11 Grossman JA, Ruchelsman DE, Schwarzkopf R. Iatrogenic spinal accessory nerve injury in children. J Pediatr Surg 2008;43(09): $1732-1735$

12 Lim JY, Lee JS, Mun BM, Kim TH. A comparison of trapezius muscle activities of different shoulder abduction angles and rotation conditions during prone horizontal abduction.J Phys Ther Sci 2015;27(01):97-100

13 Chan GY, Nonato LG, Chu A, Raghavan P, Aluru V, Silva CT. Motion Browser: visualizing and understanding complex upper limb movement under obstetrical brachial plexus injuries. IEEE Trans Vis Comput Graph 2019;26(01):981-990

14 Bertelli JA, Ghizoni MF. Transfer of the accessory nerve to the suprascapular nerve in brachial plexus reconstruction. J Hand Surg Am 2007;32(07):989-998

15 Emamhadi M, Alijani B, Andalib S. Long-term clinical outcomes of spinal accessory nerve transfer to the suprascapular nerve in patients with brachial plexus palsy. Acta Neurochir (Wien) 2016; 158(09):1801-1806

16 Garg R, Merrell GA, Hillstrom HJ, Wolfe SW. Comparison of nerve transfers and nerve grafting for traumatic upper plexus palsy: a systematic review and analysis. J Bone Joint Surg Am 2011;93 (09):819-829

17 Tung TH, Mackinnon SE. Nerve transfers: indications, techniques, and outcomes. J Hand Surg Am 2010;35(02):332-341

18 Bahm J. Upper limb multifactorial movement analysis in brachial plexus birth injury. J Brachial Plex Peripher Nerve Inj 2016;11 (01):e1-e9 
19 Heuberer P, Kranzl A, Laky B, Anderl W, Wurnig C. Electromyographic analysis: shoulder muscle activity revisited. Arch Orthop Trauma Surg 2015;135(04):549-563

20 Raghavan P, Santello M, Gordon AM, Krakauer JW. Compensatory motor control after stroke: an alternative joint strategy for objectdependent shaping of hand posture. J Neurophysiol 2010;103 (06):3034-3043

21 Sheffler LC, Lattanza L, Sison-Williamson M, James MA. Biceps brachii long head overactivity associated with elbow flexion contracture in brachial plexus birth palsy. J Bone Joint Surg Am 2012;94(04):289-297
22 Tse R, Marcus JR, Curtis CG, Dupuis A, Clarke HM. Suprascapular nerve reconstruction in obstetrical brachial plexus palsy: spinal accessory nerve transfer versus C5 root grafting. Plast Reconstr Surg 2011;127(06):2391-2396

23 Abdouni YA, Checoli GF, Salles HC, da Costa AC, Chakkour I, Fucs PMMB. Assessment of the results of accessory to suprascapular nerve transfer. Acta Ortop Bras 2018;26(05):332-334

24 Ruchelsman DE, Ramos LE, Alfonso I, Price AE, Grossman A, Grossman JA. Outcome following spinal accessory to suprascapular (spinoscapular) nerve transfer in infants with brachial plexus birth injuries. Hand (N Y) 2010;5(02):190-194 